Supporting Information

\title{
A Scalable Route to Trisubstituted (E)-Vinyl Bromides
}

Cheon-Gyu Cho ${ }^{*, \dagger}$, Won-Suk Kim and Amos B. Smith, III*,

${ }^{\dagger}$ Department of Chemistry, Hanyang University, Seoul, Korea 133-791.

${ }^{\ddagger}$ Department of Chemistry, University of Pennsylvania, Philadelphia, PA 19104, U.S.A. 
Materials and Methods. Reactions were carried out in oven or flame-dried glassware under an argon atmosphere, unless otherwise noted. All solvents were reagent grade. Diethyl ether $\left(\mathrm{Et}_{2} \mathrm{O}\right)$ and tetrahydrofuran (THF) were freshly distilled from sodium/benzophenone under argon. $n$-Butyllithium and $t$ butyllithium were purchased from Aldrich. Reactions were magnetically stirred and monitored by thin layer chromatography (TLC) with $0.25 \mathrm{~mm}$ E. Merck pre-coated silica gel plates. Flash chromatography was performed with silica gel 60 (particle size $0.040-0.062 \mathrm{~mm}$ ) supplied by Silicycle and Sorbent Technologies. Yields refer to chromatographically and spectroscopically pure compounds, unless otherwise stated. All melting points were determined on a Thomas-Hoover apparatus and are uncorrected. Infrared spectra were recorded on a Jasco Model FT/IR-480 Plus spectrometer. Proton and carbon-13 NMR spectra were recorded on a Bruker AMX-500 spectrometer. Chemical shifts are reported relative to chloroform $(\delta 7.26)$ for ${ }^{1} \mathrm{H}$ NMR and chloroform $(\delta 77.0)$ for ${ }^{13} \mathrm{C}$ NMR. Optical rotations were measured on a Perkin-Elmer model 241 polarimeter. High resolution mass spectra were measured at the University of Pennsylvania Mass Spectrometry Service Center.

\section{Experimental Procedures}
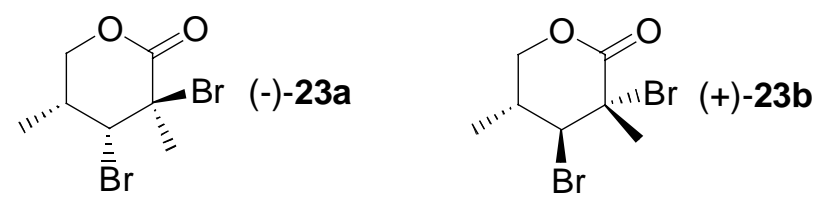

3,4-Dibromo-3,5-dimethyl-tetrahydro-pyran-2-one [(-)-23a and (+)-23b]. To a solution of (+)-22 (3.7 g, $29.3 \mathrm{mmol}$ ) in $20 \mathrm{~mL}$ of $\mathrm{CH}_{2} \mathrm{Cl}_{2}$ was added $\mathrm{Br}_{2}$ (4.6 mL, 3 equiv) slowly over $10 \mathrm{~min}$ at $-10{ }^{\circ} \mathrm{C}$ (wet ice/acetone bath). After $2 \mathrm{~h}$ at $-10^{\circ} \mathrm{C}$, the reaction mixture was slowly poured into a beaker containing 30 $\mathrm{mL}$ of sat. sodium bisulfite and $30 \mathrm{~g}$ of ice with aid of EtOAc with vigorous stirring (the quenching is quite exothermic). The organic layer was separated, washed with brine, concentrated and chromatographed (9:1 hexanes:EtOAc) to afford (-)-23a and $(+)-23 \mathbf{b}$ in the ratio of 3.7 to 1 , both as clear oils that slowly 
solidified upon standing to yield crystalline needles (total $5.9 \mathrm{~g}, 71 \%$ yield, well separated on column, but need not be collected separately).

Dibromide (-)-23a: $\mathrm{mp} 65-66{ }^{\circ} \mathrm{C}$; $[\alpha]_{\mathrm{D}}^{23}-11.6$ (c = 3.4, $\left.\mathrm{CHCl}_{3}\right)$; IR $(\mathrm{NaCl}) 2977.6,2935.1,1739.5$, 1461.8, 1380.8, 1338.4, $1261.2 \mathrm{~cm}^{-1} ;{ }^{1} \mathrm{H}$ NMR $\left(500 \mathrm{MHz}, \mathrm{CDCl}_{3}\right) \delta 4.51(\mathrm{~d}, J=1.9 \mathrm{~Hz}, 1 \mathrm{H}), 4.37-4.32$ (m, 2H), $3.31-3.24(\mathrm{~m}, 1 \mathrm{H}), 2.20(\mathrm{~s}, 3 \mathrm{H}), 1.07(\mathrm{~d}, J=6.4 \mathrm{~Hz}, 3 \mathrm{H}) ;{ }^{13} \mathrm{C} \mathrm{NMR}\left(125 \mathrm{MHz}, \mathrm{CDCl}_{3}\right) \delta 166.9$, 72.0, 63.5, 55.9, 30.5, 30.1, 15.1; high resolution mass spectrum $\left(\mathrm{Cl}^{+}\right) \mathrm{m} / \mathrm{z} 284.9131,\left[(\mathrm{M}+\mathrm{H})^{+}\right.$, calcd for $\left.\mathrm{C}_{7} \mathrm{H}_{11} \mathrm{Br}_{2} \mathrm{O}_{2}: 284.9126\right]$.

ORTEP representation of the X-ray structure of (-)-23a.

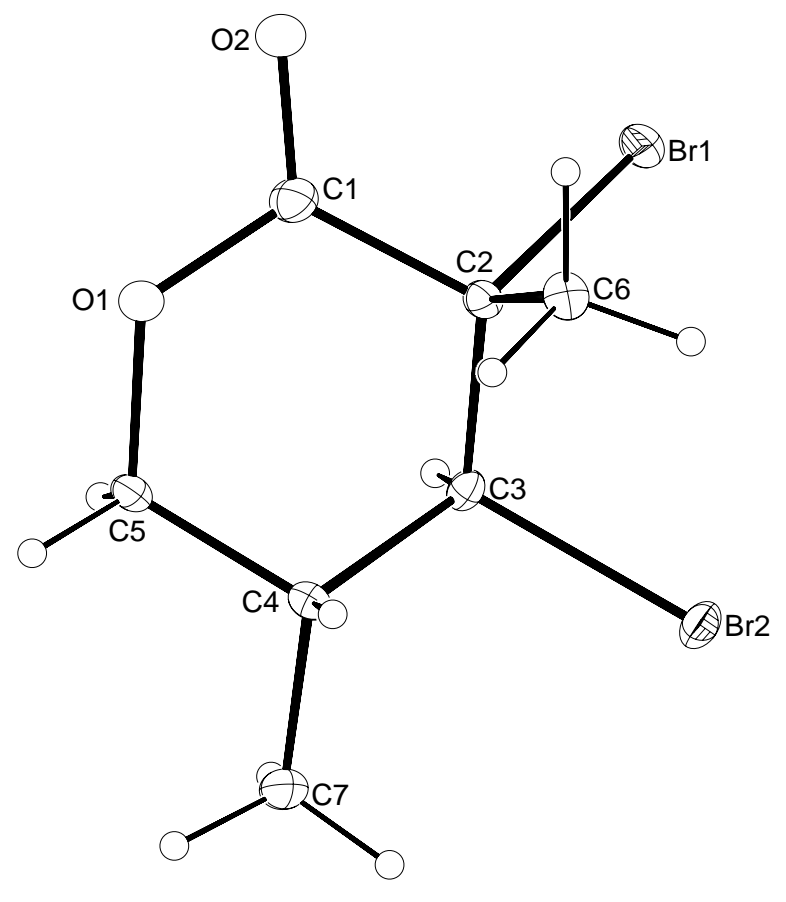


Dibromide (+)-23b: $\mathrm{mp} 85-87^{\circ} \mathrm{C} ;[\alpha]_{\mathrm{D}}^{23}+11.3\left(\mathrm{c}=2.6, \mathrm{CHCl}_{3}\right) ; \mathrm{IR}(\mathrm{NaCl}) 2973.1,2931.3,1747.2$, 1457.9, 1384.6, $1311.3 \mathrm{~cm}^{-1} ;{ }^{1} \mathrm{H}$ NMR $\left(500 \mathrm{MHz}, \mathrm{CDCl}_{3}\right) \delta 4.51(\mathrm{~d}, \mathrm{~J}=8.3 \mathrm{~Hz}, 1 \mathrm{H}), 4.40-4.38(\mathrm{~m}, 2 \mathrm{H})$, $2.71-2.62(\mathrm{~m}, 1 \mathrm{H}), 2.15(\mathrm{~s}, 3 \mathrm{H}), 1.23(\mathrm{~d}, J=6.8 \mathrm{~Hz}, 3 \mathrm{H}) ;{ }^{13} \mathrm{C} \mathrm{NMR}\left(125 \mathrm{MHz}, \mathrm{CDCl}_{3}\right) ; \delta$ 167.0, 71.7, 62.0, 56.4, 37.9, 30.5, 16.8; high resolution mass spectrum $\left(\mathrm{Cl}^{+}\right) \mathrm{m} / \mathrm{z} 284.9125,\left[(\mathrm{M}+\mathrm{H})^{+}\right.$, calcd for $\left.\mathrm{C}_{7} \mathrm{H}_{11} \mathrm{Br}_{2} \mathrm{O}_{2}: 284.9126\right]$.

ORTEP representation of the X-ray structure of $(+)-\mathbf{2 3 b}$.

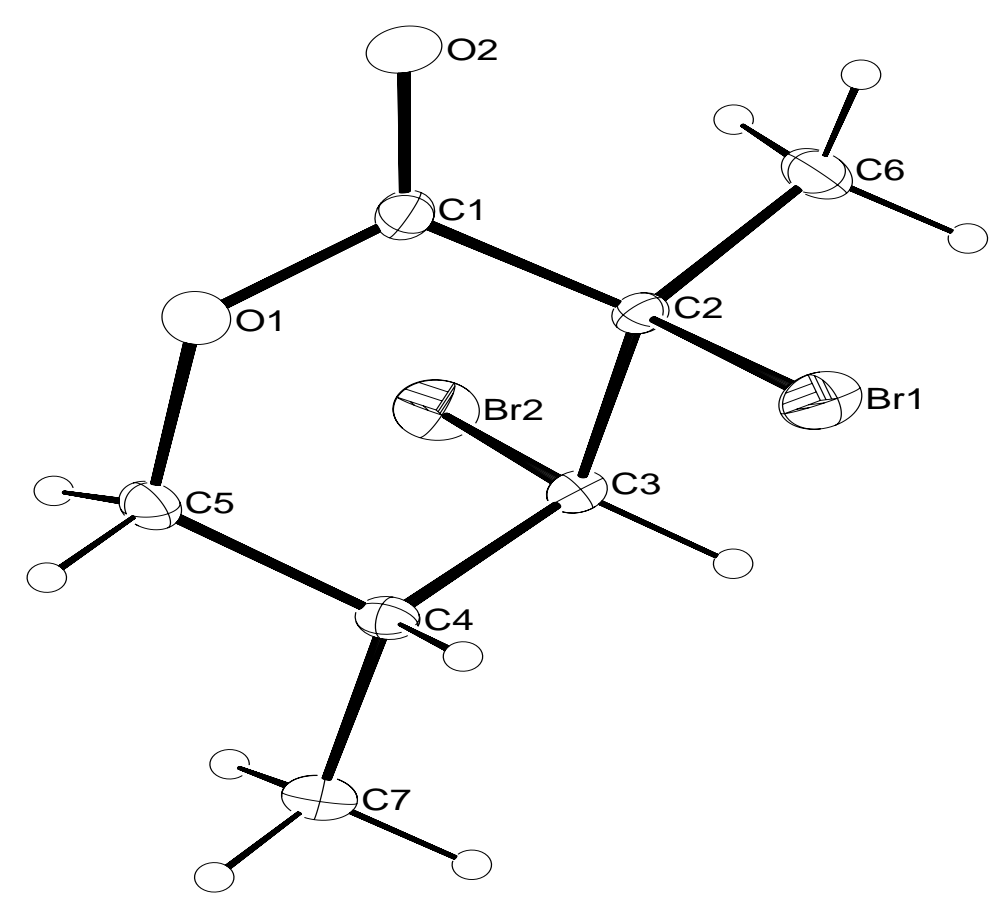




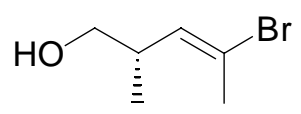

$(-)-24$

4-Bromo-2-methyl-pent-3-en-1-ol [(-)-24]. To a flask charged with (-)-23a and (+)-23b (12.6 g, 44.0 mmol) were added $100 \mathrm{~mL}$ of $\mathrm{DMF} / \mathrm{H}_{2} \mathrm{O}(4: 1)$ and $\mathrm{LiOH}$ monohydrate (5.3 g, 3 equiv) at rt. After $16 \mathrm{~h}$, the reaction mixture was directly chromatographed $\left(9: 1 \rightarrow 7: 3\right.$ pentane/ $\left.\mathrm{Et}_{2} \mathrm{O}\right)$ to furnish $(-)-24(5.8 \mathrm{~g}, 73 \%$ yield) as an essentially colorless liquid: $[\alpha]_{D}^{23} \quad-25.3\left(\mathrm{c}=2.6, \mathrm{CHCl}_{3}\right)$; IR $(\mathrm{NaCl}) 3351.7,2962.1,2927.4$, 2873.4, 1704.8, 1650.8, 1454.1, $1380.8 \mathrm{~cm}^{-1} ;{ }^{1} \mathrm{H} \mathrm{NMR}\left(500 \mathrm{MHz}, \mathrm{CDCl}_{3}\right) \delta 5.66(\mathrm{dd}, J=9.9,1.1 \mathrm{~Hz}, 1 \mathrm{H})$, $3.50(\mathrm{dd}, J=10.6,5.8 \mathrm{~Hz}, 1 \mathrm{H}), 3.41(\mathrm{dd}, J=10.6,7.7 \mathrm{~Hz}, 1 \mathrm{H}), 2.64-2.55(\mathrm{~m}, 1 \mathrm{H}), 2.27(\mathrm{~d}, J=1.1 \mathrm{~Hz}$, 3H), $0.98(\mathrm{~d}, \mathrm{~J}=6.9 \mathrm{~Hz}, 3 \mathrm{H}) ;{ }^{13} \mathrm{C} \mathrm{NMR}\left(125 \mathrm{MHz}, \mathrm{CDCl}_{3}\right) \delta 134.6,121.2,67.2,37.7,23.8,16.5$; high resolution mass spectrum: decomposition.

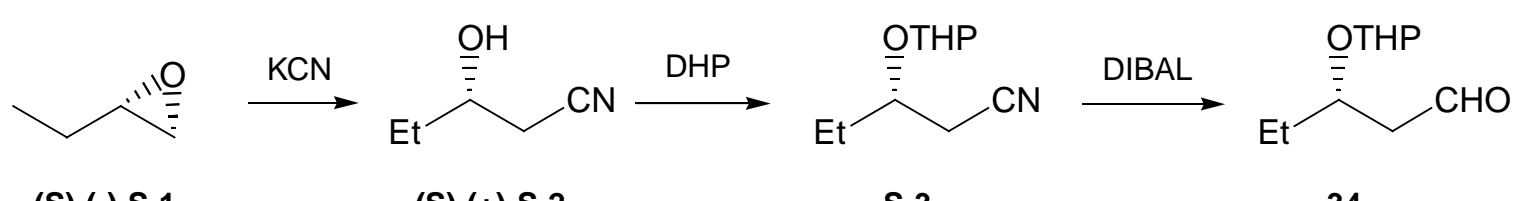

(S)-(-)-S-1

(S)-(+)-S-2

S-3

34

(S)-3-Hydroxypentanenitrile [(+)-S-2]. To a solution of (S)-1,2-epoxybutane [(-)-S-1, $6.0 \mathrm{~mL}, 69.6 \mathrm{mmol}]$ in $50 \mathrm{~mL}$ anhydrous $\mathrm{CH}_{3} \mathrm{CN}$ were added $\mathrm{KCN}(9.0 \mathrm{~g}, 138 \mathrm{mmol})$ and $\mathrm{LiClO}_{4}(14.8 \mathrm{~g}, 139 \mathrm{mmol})$ at rt. The reaction mixture was sealed and heated to $70{ }^{\circ} \mathrm{C}$. After overnight at $70{ }^{\circ} \mathrm{C}$, the reaction mixture was diluted with EtOAc and washed with saturated $\mathrm{NaHCO}_{3}$, water, and brine, dried over $\mathrm{MgSO}_{4}$, filtered and concentrated. Purification by flash chromatography (2:1 hexanes/EtOAc) afforded $6.0 \mathrm{~g}(87 \%$ yield) of (S)(-)-3-hydroxypentanenitrile [(+)-S-2] as a liquid, with spectral data matching that previously reported. ${ }^{1}$

3-(Tetrahydro-2H-pyran-2-yloxy)pentanal (34). To a solution of (+)-S-2 $(5.7 \mathrm{~g}, 57.5 \mathrm{mmol})$ in $100 \mathrm{~mL}$ of $\mathrm{CH}_{2} \mathrm{Cl}_{2}$ was added 3,4-dihydro-2H-pyran (DHP, $9.8 \mathrm{~g}, 116 \mathrm{mmol}$ ), and pyridinium p-toluenesulfonate (PPTS, $2.9 \mathrm{~g}, 11.6 \mathrm{mmol}$ ) at rt. After $2 \mathrm{~h}$ at $\mathrm{rt}$, the reaction mixture was diluted with $\mathrm{Et}_{2} \mathrm{O}(150 \mathrm{~mL})$ and 
washed with half-saturated brine $(50 \mathrm{~mL})$ to remove the catalyst. The organic layer was separated and washed with saturated $\mathrm{NaHCO}_{3}$, water, and brine, dried over $\mathrm{Na}_{2} \mathrm{SO}_{4}$, concentrated and distilled (105-106 ${ }^{\circ} \mathrm{C}$ at $\left.0.1 \mathrm{mmHg}\right)$ to give $10.4 \mathrm{~g}(99 \%$ yield $)$ of S-3 (1:1 mixture of diastereomers) as a colorless liquid: IR ( $\mathrm{NaCl})$ 2942.8, 2877.3, 2248.6, 1457.9, $1349.9 \mathrm{~cm}^{-1} ;{ }^{1} \mathrm{H}$ NMR $\left(500 \mathrm{MHz}, \mathrm{CDCl}_{3}\right) \delta 4.77$ (dd, $J=4.0,3.5$ $\mathrm{Hz}, 1 \mathrm{H}), 4.68(\mathrm{dd}, J=4.1,3.6 \mathrm{~Hz}, 1 \mathrm{H}), 3.97-3.93(\mathrm{~m}, 1 \mathrm{H}), 3.91-3.86(\mathrm{~m}, 2 \mathrm{H}), 3.80-3.75(\mathrm{~m}, 1 \mathrm{H}), 3.55$ - $3.50(\mathrm{~m}, 2 \mathrm{H}), 2.67(\mathrm{~s}, 1 \mathrm{H}), 2.66(\mathrm{~s}, 1 \mathrm{H}), 2.55(\mathrm{dd}, J=16.7,5.2 \mathrm{~Hz}, 1 \mathrm{H}), 2.48(\mathrm{dd}, J=16.7,5.6 \mathrm{~Hz}, 1 \mathrm{H})$, $1.90-1.50(\mathrm{~m}, 16 \mathrm{H}), 0.99(\mathrm{t}, J=7.4 \mathrm{~Hz}, 3 \mathrm{H}), 0.95(\mathrm{t}, J=7.4 \mathrm{~Hz}, 3 \mathrm{H}) ;{ }^{13} \mathrm{C} \mathrm{NMR}\left(125 \mathrm{MHz}, \mathrm{CDCl}_{3}\right) \delta 117.5$, 117.2, 99.2, 97.4, 74.5, 72.6, 62.7, 62.6, 30.7, 30.6, 28.0, 26.7, 25.2, 23.9, 22.1, 19.4, 19.3, 9.5, 9.2; high resolution mass spectrum $\left(\mathrm{Cl}^{+}\right) \mathrm{m} / \mathrm{z}$ 184.1334, $\left[(\mathrm{M}+\mathrm{H})^{+}\right.$, calcd for $\mathrm{C}_{10} \mathrm{H}_{18} \mathrm{NO}_{2}$ 184.1337]. To a solution of S$3(6.9 \mathrm{~g}, 37.6 \mathrm{mmol})$ in $200 \mathrm{~mL}$ of $\mathrm{CH}_{2} \mathrm{Cl}_{2}$ at $-78{ }^{\circ} \mathrm{C}$ was added DIBAL in hexanes $(1.0 \mathrm{M}, 75.0 \mathrm{~mL}, 75.0$ $\mathrm{mmol}$ ) via syringe over $30 \mathrm{~min}$. After being stirred for $3 \mathrm{~h}$, the reaction was quenched by slowly adding $\mathrm{MeOH}$ at $-78{ }^{\circ} \mathrm{C}$ followed by saturated Rochelle salts solution $\left(\mathrm{Na}^{+} / \mathrm{K}^{+}\right.$tartrate) and allowing the mixture to warm to rt. After vigorous stirring for $1 \mathrm{~h}$, the $\mathrm{CH}_{2} \mathrm{Cl}_{2}$ layer was collected, and the aqueous phase was washed with brine, dried over $\mathrm{MgSO}_{4}$, and concentrated. The crude was purified by flash chromatography (10:1 hexanes/EtOAc) to give $5.8 \mathrm{~g}$ (83\% yield) of $\mathbf{3 4}(1: 1$ mixture of diastereomers) as a liquid: IR ( $\mathrm{NaCl})$ 2942.8, 2873.4, 2730.7, 1724.1, 1457.9, $1384.6 \mathrm{~cm}^{-1} ;{ }^{1} \mathrm{H}$ NMR (500 MHz, $\left.\mathrm{CDCl}_{3}\right) \delta 9.81-9.79(\mathrm{~m}, 2 \mathrm{H})$, $4.69(\mathrm{dd}, J=4.1,3.9 \mathrm{~Hz}, 1 \mathrm{H}), 4.65(\mathrm{dd}, J=4.8,3.0 \mathrm{~Hz}, 1 \mathrm{H}), 4.12-4.06(\mathrm{~m}, 1 \mathrm{H}), 3.91-3.79(\mathrm{~m}, 2 \mathrm{H})$, $3.51-3.43(\mathrm{~m}, 2 \mathrm{H}), 2.69-2.49(\mathrm{~m}, 3 \mathrm{H}), 1.80-1.51(\mathrm{~m}, 18 \mathrm{H}), 0.96(\mathrm{t}, J=7.4 \mathrm{~Hz}, 3 \mathrm{H}), 0.91(\mathrm{t}, J=7.4 \mathrm{~Hz}$, $3 \mathrm{H}) ;{ }^{13} \mathrm{C}$ NMR $\left(125 \mathrm{MHz}, \mathrm{CDCl}_{3}\right) \delta 202.1,201.6,98.5,98.0,73.7,73.6$ 63.0, 62.8, 48.8, 47.7, 31.0, 28.6, 27.2, 25.4, 25.3, 19.9, 19.7, 9.8, 9.3; high resolution mass spectrum $\left(\mathrm{Cl}^{+}\right) \mathrm{m} / \mathrm{z} 187.1337,\left[(\mathrm{M}+\mathrm{H})^{+}\right.$, calcd for $\mathrm{C}_{10} \mathrm{H}_{19} \mathrm{O}_{3}$ 187.1334]. 


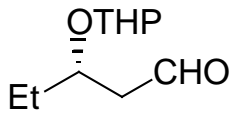

34
1) $\mathrm{EtCO}_{2} \mathrm{Et}$, LDA

2) $\mathrm{TsOH}, \mathrm{PhH}$, reflux $57 \%$ (2 steps)

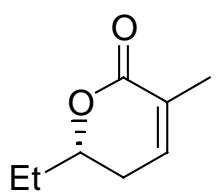

$(-)-35$

6-Ethyl-5,6-dihydro-3-methylpyran-2-one [(-)-35]. To a solution of diisopropylamine (DIPA, $10.5 \mathrm{~mL}$, $74.7 \mathrm{mmol})$ in $50 \mathrm{~mL}$ of THF at $-15{ }^{\circ} \mathrm{C}$ was added dropwise $n$-BuLi in hexanes $(2.5 \mathrm{M}, 29.9 \mathrm{~mL}, 74.7$ mmol) via syringe over $20 \mathrm{~min}$. After the addition, the reaction mixture was stirred for 15 min and then cooled to $-78^{\circ} \mathrm{C}$. To this solution was slowly added ethyl propionate $(7.2 \mathrm{~mL}, 62.3 \mathrm{mmol})$ via syringe over $10 \mathrm{~min}$. The resulting solution was stirred for $1 \mathrm{~h}$ at $-78{ }^{\circ} \mathrm{C}$. Then, aldehyde $34(5.8 \mathrm{~g}, 31.1 \mathrm{mmol})$ was added dropwise to the vigorously stirring solution at $-78{ }^{\circ} \mathrm{C}$ over $15 \mathrm{~min}$. After the solution was stirred for 30 min, saturated $\mathrm{NH}_{4} \mathrm{Cl}$ was added, and the mixture was allowed to warm to rt. The phases were separated, and the aqueous layer was extracted with $\mathrm{CH}_{2} \mathrm{Cl}_{2}$. The combined organic layers were washed with $\mathrm{H}_{2} \mathrm{O}$ and brine, dried over $\mathrm{MgSO}_{4}$, and concentrated. The crude aldol adduct (1.0 equiv) and $p$ toluenesulfonic acid (1.1 equiv) in benzene $(150 \mathrm{~mL})$ was heated to reflux for $12 \mathrm{~h}$. The water generated was trapped by means of a Dean-Stark apparatus. The reaction mixture was allowed to cool to rt, washed with $10 \% \mathrm{NaHCO}_{3}$, water, brine, dried over $\mathrm{Na}_{2} \mathrm{SO}_{4}$, concentrated and distilled $\left(90-93{ }^{\circ} \mathrm{C}\right.$ at $0.1 \mathrm{mmHg}$ ) to give $2.5 \mathrm{~g}(57 \%$ yield for 2 steps $)$ of $(-)-35$ as a liquid: $[\alpha]_{\mathrm{D}}^{23}-0.8\left(\mathrm{c}=1.4, \mathrm{CHCl}_{3}\right) ; \mathrm{IR}(\mathrm{NaCl}) 2969.8$, 2931.3, 1716.3, 1454.7, $1376.9 \mathrm{~cm}^{-1} ;{ }^{1} \mathrm{H}$ NMR $\left(500 \mathrm{MHz}, \mathrm{CDCl}_{3}\right) \delta 6.55$ (bs, $\left.1 \mathrm{H}\right), 4.31-4.25(\mathrm{~m}, 1 \mathrm{H})$, $2.27-2.25(\mathrm{~m}, 2 \mathrm{H}), 1.88(\mathrm{~s}, 3 \mathrm{H}), 1.81-1.72(\mathrm{~m}, 1 \mathrm{H}), 1.70-1.61(\mathrm{~m}, 1 \mathrm{H}), 0.98(\mathrm{t}, J=7.4 \mathrm{~Hz}, 3 \mathrm{H}) ;{ }^{13} \mathrm{C}$ $\operatorname{NMR}\left(125 \mathrm{MHz}, \mathrm{CDCl}_{3}\right) \delta 166.1,138.8,128.3,79.2,29.2,27.8,16.9,9.2$; high resolution mass spectrum $\left(\mathrm{Cl}^{+}\right) \mathrm{m} / \mathrm{z}$ 141.0919, $\left[(\mathrm{M}+\mathrm{H})^{+}\right.$, calcd for $\mathrm{C}_{8} \mathrm{H}_{13} \mathrm{O}_{2}$ 141.0915].

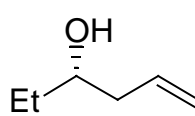

$(-)-31$

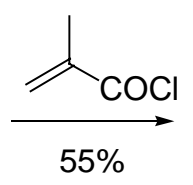

E

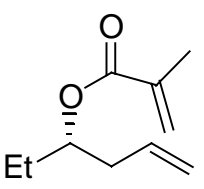

$(-)-32$

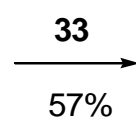

$57 \%$

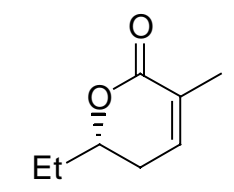

$(-)-35$ 
Hex-5-en-3-yl methacrylate [(-)-32]. To a stirred solution of (-)-31 (1.5 g, $14.9 \mathrm{mmol})$ in $20 \mathrm{~mL}$ of anhydrous $\mathrm{CH}_{2} \mathrm{Cl}_{2}$ at $0{ }^{\circ} \mathrm{C}$ was slowly added $\mathrm{Et}_{3} \mathrm{~N}(6.3 \mathrm{~mL}, 44.9 \mathrm{mmol})$, and a catalytic amount of DMAP $(0.3 \mathrm{~g}, 2.25 \mathrm{mmol})$. Methacryloyl chloride $(3.1 \mathrm{~mL}, 32.2 \mathrm{mmol})$ was then added dropwise. After removal of the cooling bath, the mixture was stirred overnight at $\mathrm{rt}$, and then quenched by the addition of saturated aqueous $\mathrm{NH}_{4} \mathrm{Cl}(20 \mathrm{~mL})$ solution. The aqueous layer was extracted with $\mathrm{CH}_{2} \mathrm{Cl}_{2}$. The organic layers were combined, washed with a saturated brine, dried over $\mathrm{MgSO}_{4}$, filtered, concentrated and purified by flash chromatography $\left(100: 1\right.$ hexanes: $\left.\mathrm{Et}_{2} \mathrm{O}\right)$ to provide $(-)-32(1.4 \mathrm{~g}, 55 \%$ yield $)$ as a liquid: $[\alpha]_{\mathrm{D}}^{23}-25.3(\mathrm{c}=$ 0.6, $\left.\mathrm{CHCl}_{3}\right) ; \mathrm{IR}(\mathrm{NaCl}) 2969.3,2935.8,1717.6,1638.2,1453.8,1380.4,1319.0,1296.3,1171.2 \mathrm{~cm}^{-1} ;{ }^{1} \mathrm{H}$ NMR (500 MHz, $\left.\mathrm{CDCl}_{3}\right) \delta 6.08$ (bs, 1H), $5.79-5.73(\mathrm{~m}, 1 \mathrm{H}), 5.51$ (bs, $\left.1 \mathrm{H}\right), 5.09-5.03(\mathrm{~m}, 2 \mathrm{H}), 4.94-$ $4.89(\mathrm{~m}, 1 \mathrm{H}), 2.35(\mathrm{t}, J=7.1 \mathrm{~Hz}, 2 \mathrm{H}), 1.93(\mathrm{~s}, 3 \mathrm{H}), 1.66-1.59(\mathrm{~m}, 2 \mathrm{H}), 0.90(\mathrm{t}, J=7.4 \mathrm{~Hz}, 3 \mathrm{H}) ;{ }^{13} \mathrm{C}$ NMR $\left(125 \mathrm{MHz}, \mathrm{CDCl}_{3}\right) \delta 167.1,136.8,133.8,124.9,117.5,74.8,38.1,26.5,18.3,9.5$; high resolution mass spectrum $\left(\mathrm{Cl}^{+}\right) \mathrm{m} / \mathrm{z}$ 169.1171, [(M+H $)^{+}$, calcd for $\mathrm{C}_{10} \mathrm{H}_{17} \mathrm{O}_{2}$ 169.1229].

6-Ethyl-5,6-dihydro-3-methylpyran-2-one [(-)-35]. To a solution of (-)-32 (107 mg, $0.64 \mathrm{mmol})$ in $\mathrm{CH}_{2} \mathrm{Cl}_{2}$ $(0.05 \mathrm{M})$ was added the ruthenium complex $33(27 \mathrm{mg}, 0.03 \mathrm{mmol})$ at $\mathrm{rt}$. The reaction mixture was heated at reflux for $14 \mathrm{~h}$. The reaction mixture was then concentrated and purified directly on a silica gel column, eluting with 10:1 (hexanes/EtOAc) to give $51 \mathrm{mg} \mathrm{(57 \%} \mathrm{yield)} \mathrm{of} \mathrm{(-)-35.}$

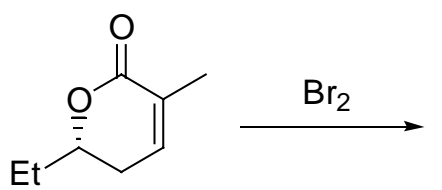

$(-)-35$<smiles>CCC1CC(Br)[C@](C)(Br)C(=O)O1</smiles>

S-4-A<smiles>CCC1CC(Br)C(C)(Br)C(=O)O1</smiles>

S-4-B

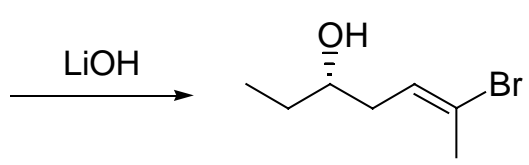

$(-)-36$

Dibromides (+)-S-4-A and S-4-B. To a solution of (-)-35 (2.4 g, $17.1 \mathrm{mmol})$ in $30 \mathrm{~mL}$ of $\mathrm{CH}_{2} \mathrm{Cl}_{2}$ was added $\mathrm{Br}_{2}(2.6 \mathrm{~mL}, 51.4 \mathrm{mmol})$ slowly over $10 \mathrm{~min}$ at $-10{ }^{\circ} \mathrm{C}$ (wet ice/acetone bath). After $3 \mathrm{~h}$ at $-10{ }^{\circ} \mathrm{C}$, the reaction mixture was slowly poured into a beaker containing $30 \mathrm{~mL}$ of saturated sodium bisulfite and $30 \mathrm{~g}$ of ice with aid of EtOAc with vigorous stirring. The organic layer was separated, washed with brine, 
concentrated and chromatographed (15:1 hexanes: $\mathrm{Et}_{2} \mathrm{O}$ ) to afford S-4-A and S-4-B (in the ratio of 4 to 1 , total $3.2 \mathrm{~g}, 62 \%$ yield): (+)-S-4-A. $[\alpha]_{\mathrm{D}}^{23}+22.0\left(\mathrm{c} 0.7, \mathrm{CHCl}_{3}\right)$; IR (NaCl) 2973.7, 2935.1, 1739.5, 1442.5, 1380.8, $1284.4 \mathrm{~cm}^{-1} ;{ }^{1} \mathrm{H}$ NMR $\left(500 \mathrm{MHz}, \mathrm{CDCl}_{3}\right) \delta 4.94-4.88(\mathrm{~m}, 1 \mathrm{H}), 4.71(\mathrm{dd}, J=2.9,2.7 \mathrm{~Hz}, 1 \mathrm{H}), 2.99$ (ddd, $J=14.1,11.5,2.7 \mathrm{~Hz}, 1 \mathrm{H}), 2.20(\mathrm{dd}, J=3.7,3.7 \mathrm{~Hz}, 1 \mathrm{H}), 2.17(\mathrm{~s}, 3 \mathrm{H}), 1.89-1.73(\mathrm{~m}, 2 \mathrm{H}), 1.05(\mathrm{t}, J$ $=7.8 \mathrm{~Hz}, 3 \mathrm{H}) ;{ }^{13} \mathrm{C} \mathrm{NMR}\left(125 \mathrm{MHz}, \mathrm{CDCl}_{3}\right) \delta 167.3,79.8,54.9,54.2,34.4,29.6,28.2,9.0$; high resolution mass spectrum $\left(\mathrm{Cl}^{+}\right) \mathrm{m} / \mathrm{z}$ 298.9280, $\left[(\mathrm{M}+\mathrm{H})^{+}\right.$, calcd for $\mathrm{C}_{8} \mathrm{H}_{13} \mathrm{O}_{2} \mathrm{Br}_{2}$ 298.9282]. S-4-B (unstable): ${ }^{1} \mathrm{H}$ NMR $\left(500 \mathrm{MHz}, \mathrm{CDCl}_{3}\right) \delta 4.81-4.76(\mathrm{~m}, 1 \mathrm{H}), 4.69(\mathrm{dd}, J=6.3,4.0 \mathrm{~Hz}, 1 \mathrm{H}), 2.93-2.87(\mathrm{~m}, 1 \mathrm{H}), 2.36(\mathrm{ddd}, J=$ 12.5, 8.5, 3.9 Hz, $1 \mathrm{H}), 2.15(\mathrm{~s}, 3 \mathrm{H}), 1.89-1.71(\mathrm{~m}, 2 \mathrm{H}), 1.05(\mathrm{t}, J=7.4 \mathrm{~Hz}, 3 \mathrm{H}) ;{ }^{13} \mathrm{C}$ NMR $(125 \mathrm{MHz}$, $\left.\mathrm{CDCl}_{3}\right) \delta 166.7,78.4,54.9,51.936 .4,29.7,29.4,9.0$; high resolution mass spectrum $\left(\mathrm{Cl}^{+}\right) \mathrm{m} / \mathrm{z} 298.9273$, $\left[(\mathrm{M}+\mathrm{H})^{+}\right.$, calcd for $\mathrm{C}_{8} \mathrm{H}_{13} \mathrm{O}_{2} \mathrm{Br}_{2}$ 298.9282].

6-Bromo-hept-5-en-3-ol [(-)-36]. To a solution To a flask charged with (+)-S-4-A and S-4-B (3.0 g, 10.0 mmol) were added $25 \mathrm{~mL}$ of $\mathrm{DMF} / \mathrm{H}_{2} \mathrm{O}(4: 1)$ and $\mathrm{LiOH}$ monohydrate $(1.3 \mathrm{~g}, 30.0 \mathrm{mmol})$ at rt. After $16 \mathrm{~h}$, the reaction mixture was directly chromatographed $\left(9: 1 \rightarrow 7: 3\right.$ pentane/ $\left.\mathrm{Et}_{2} \mathrm{O}\right)$ to furnish $(-)-36(1.3 \mathrm{~g}, 68 \%$ yield) as a liquid: $[\alpha]_{D}^{23}-1.8\left(\mathrm{c}=1.6, \mathrm{CHCl}_{3}\right)$; IR $(\mathrm{NaCl}) 3371.3,2963.4,2926.8,1649.8,1597.9,1458.7$, 1428.5, $1380.0 \mathrm{~cm}^{-1} ;{ }^{1} \mathrm{H}$ NMR $\left(500 \mathrm{MHz}, \mathrm{CDCl}_{3}\right) \delta 5.90(\mathrm{t}, J=8.8 \mathrm{~Hz}, 1 \mathrm{H}), 3.58(\mathrm{bs}, 1 \mathrm{H}), 2.23(\mathrm{~s}, 3 \mathrm{H})$, 2.22-2.12 (m, 2H), $1.65(\mathrm{~d}, J=3.0 \mathrm{~Hz}, 1 \mathrm{H}), 1.56-1.41(\mathrm{~m}, 2 \mathrm{H}), 0.95(\mathrm{t}, J=7.4 \mathrm{~Hz}, 3 \mathrm{H}) ;{ }^{13} \mathrm{C} \mathrm{NMR}(125 \mathrm{MHz}$, $\left.\mathrm{CDCl}_{3}\right) \delta 128.3,121.5,72.3,37.0,29.6,23.4,9.9$; high resolution mass spectrum $\left(\mathrm{Cl}^{+}\right) \mathrm{m} / \mathrm{z}$ 175.0115, [(M$\mathrm{OH})^{+}$, calcd for $\left.\mathrm{C}_{7} \mathrm{H}_{12} \mathrm{Br} 175.0122\right]$.

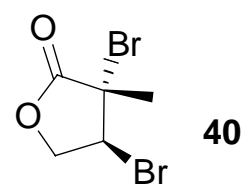

3,4-Dibromo-3-methyl-dihydro-furan-2-one (40). Under the conditions similar to those used for (+)-22, the bromination of $37(2.5 \mathrm{~g}, 25.48 \mathrm{mmol})$ gave 40 (6.1 g, 93\% yield) as a white solid: IR ( $\mathrm{NaCl}) 3023.8$, 
1785.8, 1446.4, 1376.9, $1180.2 \mathrm{~cm}^{-1} ;{ }^{1} \mathrm{H}$ NMR $\left(500 \mathrm{MHz}, \mathrm{CDCl}_{3}\right) \delta 4.98(\mathrm{dd}, J=11.7,3.7 \mathrm{~Hz}, 1 \mathrm{H}), 4.84(\mathrm{~d}$, $J=3.7 \mathrm{~Hz}, 1 \mathrm{H}), 4.53(\mathrm{~d}, J=11.1 \mathrm{~Hz}, 1 \mathrm{H}), 2.06(\mathrm{~s}, 3 \mathrm{H}) ;{ }^{13} \mathrm{CNMR}\left(125 \mathrm{MHz}, \mathrm{CDCl}_{3}\right) \delta 171.9,73.8,54.3$, 52.8, 25.3; high resolution mass spectrum $\left(\mathrm{Cl}^{+}\right) \mathrm{m} / \mathrm{z} 256.8817,\left[(\mathrm{M}+\mathrm{H})^{+}\right.$, calcd for $\left.\mathrm{C}_{5} \mathrm{H}_{7} \mathrm{Br}_{2} \mathrm{O}_{2} 256.8813\right]$.

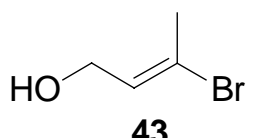

(E)-5-Bromo-but-2-en-1-ol (43). Under the conditions similar to those used for (-)-23a, decarboxylative debromination of $40(5.5 \mathrm{~g}, 21.3 \mathrm{mmol})$ gave $43(1.9 \mathrm{~g}, 59 \%$ yield) as a tan oil, upon direct chromatography $\left(9: 1 \rightarrow 7: 3\right.$ pentane/ $\left.\mathrm{Et}_{2} \mathrm{O}\right)$, with spectral data matching that previously reported. ${ }^{2}$

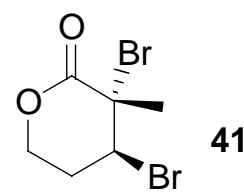

3,4-Dibromo-3-methyl-tetrahydro-pyran-2-one (41). Under conditions similar to those used for (+)-22, the bromination of $38(1.0 \mathrm{~g}, 8.9 \mathrm{mmol})$ gave 41 (1.58 g, 65\% yield) as white solids. IR ( $\mathrm{NaCl}) 2985.1$, 1737.4, 1275.9, $1164.2 \mathrm{~cm}^{-1} ;{ }^{1} \mathrm{H}$ NMR $\left(500 \mathrm{MHz}, \mathrm{CDCl}_{3}\right) \delta 4.88$ (ddd, $\left.J=16.2,11.9,4.4 \mathrm{~Hz}, 1 \mathrm{H}\right), 4.74$ (dd, $J=3.4,0.9 \mathrm{~Hz}, 1 \mathrm{H}$ ), 4.62 (qt, $J=6.6,1.3 \mathrm{~Hz}, 1 \mathrm{H}), 3.32-3.25(\mathrm{~m}, 1 \mathrm{H}), 2.17(\mathrm{~s}, 3 \mathrm{H}), 2.17-2.13(\mathrm{~m}, 1 \mathrm{H})$; ${ }^{13} \mathrm{C}$ NMR $\left(125 \mathrm{MHz}, \mathrm{CDCl}_{3}\right) \delta 166.9,67.1,55.2,54.4,29.7,29.3$; high resolution mass spectrum $\left(\mathrm{Cl}^{+}\right) \mathrm{m} / \mathrm{z}$ 270.8965, $\left[(\mathrm{M}+\mathrm{H})^{+}\right.$, calcd for $\left.\mathrm{C}_{6} \mathrm{H}_{9} \mathrm{Br}_{2} \mathrm{O}_{2} 270.8969\right]$.

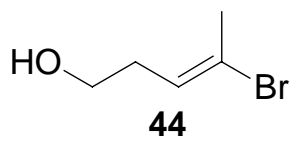

(E)-5-Bromo-but-2-en-1-ol (44). Under the conditions similar to those used for (-)-23a, decarboxylative debromination of $41(1.00 \mathrm{~g}, 3.49 \mathrm{mmol})$ gave $44(0.46 \mathrm{~g}, 80 \%$ yield $)$ as a tan oil, upon direct chromatography $\left(9: 1 \rightarrow 7: 3\right.$ pentane/Et $\left.{ }_{2} \mathrm{O}\right): \mathrm{IR}(\mathrm{NaCl}) 3243.7,2950.3,1651.9,1429.1,1379.3,1054.4 \mathrm{~cm}^{-}$ 
${ }^{1} ;{ }^{1} \mathrm{H}$ NMR $\left(500 \mathrm{MHz}, \mathrm{CDCl}_{3}\right) \delta 5.86(\mathrm{td}, J=7.7,1.1 \mathrm{~Hz}, 1 \mathrm{H}), 3.64(\mathrm{t}, J=6.4 \mathrm{~Hz}, 2 \mathrm{H}), 2.28(\mathrm{td}, J=7.7,6.5$ $\mathrm{Hz}, 2 \mathrm{H}), 2.24(\mathrm{~s}, 3 \mathrm{H}) ;{ }^{13} \mathrm{CNMR}\left(125 \mathrm{MHz}, \mathrm{CDCl}_{3}\right) \delta 128.2,121.8,61.5,33.0,23.4$; high resolution mass spectrum: decomposition.

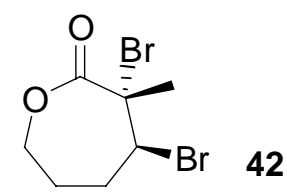

3,4-Dibromo-3-methyl-oxepan-2-one (42). Under conditions similar to those used for (+)-22, bromination of $39(0.8 \mathrm{~g}, 6.34 \mathrm{mmol})$ gave $42(1.0 \mathrm{~g}, 55 \%$ yield): IR ( $\mathrm{NaCl}) 2968.4,2938.5,1723.0,1468.8,1434.9$, 1288.2, 1226.5, $1172.8 \mathrm{~cm}^{-1} ;{ }^{1} \mathrm{H}$ NMR $\left(500 \mathrm{MHz}, \mathrm{CDCl}_{3}\right) \delta 4.97(\mathrm{t}, J=12.4 \mathrm{~Hz}, 1 \mathrm{H}), 4.45(\mathrm{t}, J=3.4 \mathrm{~Hz}$, 1H), $4.41-4.37(m, 1 H), 2.88-2.81(m, 1 H), 2.47-2.39(m, 1 H), 2.32-2.27(m, 1 H), 2.23(s, 3 H) 1.91$ - $1.86(\mathrm{~m}, 1 \mathrm{H}) ;{ }^{13} \mathrm{C} \mathrm{NMR}\left(125 \mathrm{MHz}, \mathrm{CDCl}_{3}\right) \delta 167.4,69.9,61.0,56.2,35.1,33.8,24.4$; high resolution mass spectrum $\left(\mathrm{Cl}^{+}\right) \mathrm{m} / \mathrm{z}$ 284.9113, $\left[(\mathrm{M}+\mathrm{H})^{+}\right.$, calcd for $\left.\mathrm{C}_{7} \mathrm{H}_{11} \mathrm{Br}_{2} \mathrm{O}_{2} 284.9126\right]$.

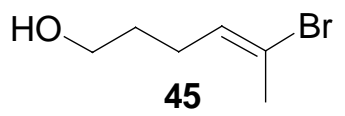

(E)-5-Bromo-hex-4-en-1-ol (45). Under the conditions similar to those used for (+)-23a, the hydrolytic fragmentation of $42(1.00 \mathrm{~g}, 3.5 \mathrm{mmol})$ gave $45(0.39 \mathrm{~g}, 62 \%$ yield) as a tan oil, upon direct chromatography $(9: 1 \rightarrow 7: 3$ pentane/Et $2 \mathrm{O})$, with spectral data matching that previously reported. ${ }^{3}$

(1) Itoh, T.; Misukura, K.; Kanphai, K.; Kanphai, W.; Takagi, Y.; Kihara, H.; Tsukube, H. J. Org. Chem. 1997, 62, 9165.

(2) Stork, G.; Baine, N. H. Tetrahedron Lett. 1985, 26, 5927.

(3) Marumoto, S.; Kogen, H.; Naruto, S. Tetrahedron 1999, 55, 7145. 